\title{
Targeted sequencing analysis of PPARG identifies a risk variant associated with obstructive sleep apnea in Chinese Han subjects
}

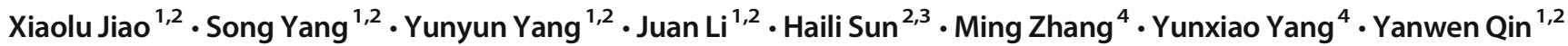

Received: 28 November 2018 /Revised: 11 April 2019 / Accepted: 23 April 2019/Published online: 2 May 2019

(C) The Author(s) 2019

\begin{abstract}
Purpose Obstructive sleep apnea (OSA) is a common disorder characterized by recurrent episodes of partial or complete upper airway obstruction. OSA susceptibility is associated with multiple genetic, environmental, and developmental factors. The PPARG rs $1801282(\mathrm{G} / \mathrm{C})$ polymorphism has been associated with OSA in obese Indian subjects, whereas no such association has been reported in Chinese Han subjects. Potential associations between other PPARG variants and OSA have not been investigated in Chinese Han populations. The aim of this study was to identify genetic variants of PPARG in unrelated Chinese Han patients with OSA and to investigate potential associations between these variants and OSA.

Methods We performed a cross-sectional study of 233 individuals with OSA and 93 control individuals. A portable diagnostic device was used to diagnose OSA. Targeted sequencing was conducted to identify PPARG variants. Associations between $P P A R G$ variants and OSA were analyzed using multivariate regression.

Results Three PPARG single-nucleotide polymorphisms were identified and the genotype frequencies of the rs 1801282 polymorphism differed significantly. Subjects with the $P P A R G$ rs 1801282 CG genotype had decreased risk of having OSA compared with subjects with the $\mathrm{CC}$ genotype after adjusting for confounding effects.

Conclusions We identified a variant of PPARG associated with the occurrence of OSA in Chinese Han populations.
\end{abstract}

Keywords Obstructive sleep apnea · Peroxisome proliferator-activated receptor gamma - Single nucleotide polymorphisms . Targeted sequencing

Electronic supplementary material The online version of this article (https://doi.org/10.1007/s11325-019-01855-x) contains supplementary material, which is available to authorized users.

Yanwen Qin

qinyanwen@vip.126.com; qinyanwen@ccmu.edu.cn

1 Key Laboratory of Remodeling-related Cardiovascular Diseases, Beijing Anzhen Hospital, Beijing Institute of Heart, Lung and Blood Vessel Diseases, Capital Medical University, No. 2 Anzhen Road, Chaoyang District, Beijing 100029, China

2 Key Laboratory of Upper Airway Dysfunction-related Cardiovascular Diseases, Beijing Anzhen Hospital, Beijing Institute of Heart, Lung and Blood Vessel Diseases, Capital Medical University, Beijing 100029, China

3 Otolaryngological Department of Beijing Anzhen Hospital, Capital Medical University, Beijing 100029, China

4 Department of Cardiology, Beijing Anzhen Hospital, Capital Medical University, Beijing 100029, China

\section{Introduction}

Obstructive sleep apnea (OSA) is a relatively common sleep disorder characterized by recurrent episodes of partial or complete collapse of the upper airway during sleep, leading to loud snoring, sleep fragmentation, daytime sleepiness, and chronic episodes of intermittent hypoxia [1]. It has been estimated that $34 \%$ of men and $17 \%$ of women are affected by OSA $[2,3]$. Patients with OSA are at high risk of hypertension, diabetes, cardiovascular diseases, stroke, and other disorders [1, 4]. Previous studies have reported multiple risk factors for OSA [5-9], including obesity, male sex, micrognathia, menopause, fluid retention, adenotonsillar hypertrophy, and smoking. However, the pathogenesis of OSA is not fully understood.

OSA clusters within families [5] and having a first-degree relative with OSA increases the risk of OSA by more than 1.5fold. The apnea-hypopnea index (AHI) is the most commonly used metric for OSA, and approximately $35 \%$ to $40 \%$ of variation in AHI can be explained by genetic factors [6, 10-13]. Other than male sex, the primary risk factor for OSA is 
excessive weight gain and obesity is the strongest risk factor for the development of OSA, and at least $70 \%$ of OSA patients are obese [14]. Peroxisome proliferator-activated receptor gamma (PPARG) is a member of the nuclear receptor family that includes 48 human transcription factors [15]. PPARG has been linked to development of obesity [15-17] and may play an important role in the pathophysiological mechanisms underlying OSA. PPARG expression was downregulated in the adipose tissue of OSA patients relative to control individuals, as measured using qPCR [18].

The PPARG rs $1801282(\mathrm{G} / \mathrm{C})$ polymorphism was reported to be associated with OSA in obese Indian subjects [19], whereas no association between rs1801282 (G/C) and OSA was observed in a Chinese Han population [20]. Potential associations between other PPARG variants and OSA have not been investigated in Chinese Han individuals.

In this study, we aimed to identify genetic variants of PPARG by targeted sequencing in unrelated Chinese Han subjects and to explore potential associations between OSA and the identified variants.

\section{Methods}

\section{Subjects}

This was a cross-sectional study. In total, 326 unrelated Chinese Han individuals aged $\geq 18$ years were recruited, including 233 patients with OSA and 93 controls. The study flow chart is shown in Supplemental Data Fig. S1. Patients who were seen in the Otolaryngological Department at Beijing Anzhen Hospital between April 2017 and November 2017 were screened for OSA using the Berlin questionnaire, which contains three categories of questions related to risk of sleep apnea: snoring and cessation of breathing, daytime sleepiness, and obesity or hypertension. Subjects are considered to be at high risk if their scores are positive in two or more categories [21] and are otherwise considered at low risk for OSA. Patients with respiratory diseases requiring medication, congestive heart failure, cancer, acute infectious diseases, hepatic dysfunction, abnormal renal function, or current pregnancies were excluded $[22,23]$. Patients with physiciandiagnosed narcolepsy, idiopathic hypersomnolence, chronic insomnia, restless legs syndrome, or any other sleep disorder were also excluded [22]. The diagnosis of narcolepsy, idiopathic hypersomnolence, chronic insomnia, restless legs syndrome, or any other sleep disorder were depended on medical histories in this study. We also excluded patients who used hypnotics, anxiolytics, sedating antidepressants, anticonvulsants, sedating antihistamines, stimulants, or other medications likely to affect the function of the nervous system [22, 23]. Finally, we selected a total of 360 eligible subjects. Each eligible subject was scheduled for an overnight sleep study, which was conducted using a level II portable diagnostic device (SOMNOscreen; SOMNOmedics GmbH, Randersacker, Germany) approved by the US Food and Drug Administration. The SOMNOscreen device assesses airflow by means of a nasal cannula pressure transducer and an oronasal thermal sensor, thoracic and abdominal movement using inductance plethysmography, and oxygen saturation by means of pulse oximetry with a fingertip sensor. Patients who were at low risk based on the Berlin questionnaire and had an $\mathrm{AHI} \geq 5$ based on the results of overnight sleep study, as well as patients who were at high risk and had an $\mathrm{AHI}<15$, were excluded. Individuals who were at low risk based on the Berlin questionnaire and had $\mathrm{AHI}<5$ were classified as controls, while individuals who were at high risk and had $\mathrm{AHI} \geq$ 15 were classified as having OSA. Finally, a total of 326 subjects were included in this study, including 233 patients with OSA and 93 controls. All participants gave written informed consent before enrollment. The protocol was approved by the Medicine Ethics Committee of Beijing Anzhen Hospital (2017005) and adhered to the principles laid out in the Declaration of Helsinki. This study is registered in the Chinese Clinical Trial Registry (No. ChiCTR-ROC17011027).

OSA was defined as having an AHI of $\geq 15$ events per hour according to the American Academy of Sleep Medicine guidelines [24-26]. In this study, the quantitative outcomes were the AHI and the lowest oxygen saturation (SpO2) across the recording period [27]. The AHI was calculated as the number of apnea and hypopnea events recorded per hour, where apnea was defined as breathing cessation for $>10 \mathrm{~s}$ and hypopnea was defined as respiratory airflow reduced by $30 \%$ with a $4 \%$ decrease in $\mathrm{SpO} 2$ [24]. Epworth Sleepiness Scale (ESS) was used to identify daytime sleepiness.

Anthropometric determinations and blood extractions were performed on a single day as described previously [28]. Blood samples were taken after overnight fasting, and routine laboratory analyses were performed to measure fasting blood glucose, lipid profiles, and other blood chemistry markers. Weight and height were measured (subjects wore light indoor clothing and were barefoot) and the body mass index (BMI) $\left(\mathrm{kg} / \mathrm{m}^{2}\right)$ was calculated using the formula: weight (kilograms) divided by height (meters) squared. According to the recommendations of the Health Promotion Administration, obesity was defined as $\mathrm{BMI} \geq 28 \mathrm{~kg} / \mathrm{m}^{2}$ [29]. Blood pressure was measured at the right upper arm after a 5-min rest in a sitting position at least three times, and the mean value was used in the analyses. Nonsmokers were subjects who had never smoked or had stopped smoking for $\geq 1$ year before enrollment in the study. All remaining subjects were classified as smokers. Drinkers were defined as subjects who consumed alcohol $\geq 3$ days a week. The definitions of dyslipidemia, diabetes, and hypertension were based on subject medical histories. 


\section{DNA template preparation and amplification}

Genomic DNA was extracted from $200 \mu \mathrm{L}$ of whole blood using a QIAamp DNA Mini Kit (Qiagen, Hilden, Germany) according to the manufacturer's protocol. The multiplex PCR amplification strategy to amplify target DNA sequences was designed online (Ion Ampliseq ${ }^{\mathrm{TM}}$ Designer; http://www. ampliseq.com). Primer sequences are listed in Supplemental Data Table S1. After evaluating the primer design options, the design that provided the maximum coverage was chosen. The amplicons covered approximately $99.80 \%$ of the target sequence. Ten nanograms of DNA per sample was used for enrichment by multiplex PCR. Each DNA pool was amplified using an Ion Ampliseq ${ }^{\mathrm{TM}}$ Library Kit in conjunction with the Ion Ampliseq ${ }^{\mathrm{TM}}$ Custom Primer Pool according to the manufacturer's protocols (Life Technologies, Darmstadt, Germany).

After each pool had undergone 17 PCR cycles, the PCR primers were removed with FuPa Reagent (Life Technologies) and the amplicons were ligated to sequencing adaptors containing short barcodes that enabled sample multiplexing using an Ion Xpress ${ }^{\mathrm{TM}}$ Barcode Adapters Kit (Life Technologies). After purification with AMPure XP beads (Beckman Coulter, Krefeld, Germany), the barcoded libraries were quantified with a Qubit ${ }^{2}$ 2.0 Fluorimeter (Life Technologies) and the DNA concentration was normalized to a final concentration of $20 \mathrm{pmol} / \mathrm{L}$ using an Ion Library Equalizer ${ }^{\mathrm{TM}} \mathrm{Kit}$ (Life Technologies). Equimolar amounts of barcoded libraries from 64 samples at a time were pooled. To clonally amplify the library DNA onto Ion Sphere Particles (Life Technologies), the library pool was subjected to emulsion PCR using an IT OneTouch Template kit on an IT OneTouch system (Life Technologies) following the manufacturer's protocol.

\section{Targeted sequencing}

The PPARG sequence was obtained using Ion Torrent semiconductor sequencing (Life Technologies, Carlsbad, CA, USA). Enriched Ion Sphere Particles that carried many copies of the same DNA fragment were sequenced on an Ion 318 Chip to sequence pooled libraries containing 64 samples at a time. Sequencing was performed using an Ion PGM Sequencing kit (Life Technologies) with the 400-bp singleend run configuration according to the manufacturer's instructions. Details of the primers used are given in Supplemental Data Table S2.

\section{Computational analysis}

Torrent Suite software version 4.4.2 (Life Technologies, Darmstadt, Germany) was used to align reads from the raw data (unmapped BAM files). The read alignments were filtered by Torrent Suite into mapped BAM files using the human reference genomic sequence (hg19) of PPARG. Variant calling was performed with the Torrent Variant Caller Plugin (minimum allele frequency 0.2 ; minimum quality 20 ; minimum coverage 20 ; and minimum coverage on either strand 3). Annotation of variants was performed using Ion Reporter software version 4.4 (Life Technologies, Darmstadt, Germany) for the variant call format files. The annotations included genomic and complementary DNA positions, genetic reference sequences, amino acid changes, and related information available from public databases such as the 1000 Genomes Project, the National Heart, Lung, and Blood Institute Grand Opportunity Exome Sequencing Project (ESP6500) (https://esp.gs.washington.edu/drupal/), the Single Nucleotide Polymorphism Database (dbSNP147) (National Center for Biotechnology Information, http://www. ncbi.nlm.nih.gov/SNP/), the Exome Aggregation Consortium (ExAC03) (http://exac.broadinstitute.org), ClinVar, DrugBank, Online Mendelian Inheritance in Man (OMIM), and the Human Gene Mutation Database (HGMD).

\section{Statistical analysis}

Continuous variables were expressed as means \pm standard deviations (for normally distributed data) or medians (interquartile ranges) (for asymmetrically distributed data) and categorical variables were expressed as numerals (percentages). Independent Student's $t$ tests (for normally distributed data) and Wilcoxon rank sum tests (for asymmetrically distributed data) were used to analyze differences in continuous variables. Chi-squared tests and Fisher's exact tests were used to analyze categorical variables. Deviations of genotype frequencies from the assumption of Hardy-Weinberg equilibrium were assessed using a chi-square test. Associations between OSA and PPARG variants were analyzed by logistic regression analysis. Step-down Bonferroni correction was used to adjust for multiple comparisons in exploring the association between OSA and PPARG variants. All probability values were twosided, and a $P$ value $<0.05$ was considered statistically significant. All analyses were performed with R (http://www.Rproject.org) and Empower Stats software (www. empowerstats.com; X\&Y Solutions, Inc., Boston, MA, USA).

\section{Results}

\section{Baseline clinical characteristics of the study population}

The clinical characteristics of the 233 individuals with OSA and 93 controls who participated in this study are shown in Table 1. There were no significant differences in age $(P=$ $0.122)$, systolic blood pressure $(P=0.091)$, total cholesterol $(P=0.585)$, low-density lipoprotein cholesterol $(P=0.636)$, 
Table 1 Anthropometric and biochemical characteristics of the subjects included in the study

\begin{tabular}{|c|c|c|c|}
\hline & OSAHS $N=233$ & Non-OSAHS $N=93$ & $P$ value \\
\hline Age (years) & $55.29 \pm 10.47$ & $52.82 \pm 13.79$ & 0.122 \\
\hline Male $(\mathrm{n}, \%)$ & $200(85.84 \%)$ & $66(70.97 \%)$ & $0.002 *$ \\
\hline BMI $\left(\mathrm{kg} / \mathrm{m}^{2}\right)$ & $27.52 \pm 3.50$ & $24.02 \pm 3.58$ & $<0.001 * *$ \\
\hline Obesity $(\mathrm{n}, \%)$ & $99(42.49 \%)$ & $15(16.13 \%)$ & $<0.001 * *$ \\
\hline Smoker $(\mathrm{n}, \%)$ & $102(43.78 \%)$ & $27(29.03 \%)$ & $0.009 *$ \\
\hline Drinker (n, \%) & $98(42.06 \%)$ & $35(37.63 \%)$ & 0.276 \\
\hline Hypertension (yes, N n,\%) & $59(25.32 \%)$ & $12(12.90 \%)$ & $0.009 *$ \\
\hline Diabetes (yes, N n,\%) & $16(6.87 \%)$ & $5(5.38 \%)$ & 0.340 \\
\hline Dyslipidemia (yes, N n,\%) & $175(75.11 \%)$ & $54(58.06 \%)$ & $0.002 *$ \\
\hline $\mathrm{SBP}(\mathrm{mmHg})$ & $126.45 \pm 18.49$ & $122.66 \pm 17.56$ & 0.091 \\
\hline $\mathrm{DBP}(\mathrm{mmHg})$ & $77.31 \pm 12.38$ & $74.10 \pm 11.66$ & $0.032 *$ \\
\hline FBG (mmol/L) & $4.67 \pm 1.40$ & $4.36 \pm 2.03$ & 0.201 \\
\hline TG (mmol/L) & $1.59(1.12-2.18)$ & $1.27(0.94-1.62)$ & $<0.001 * *$ \\
\hline $\mathrm{TC}(\mathrm{mmol} / \mathrm{L})$ & $4.31 \pm 1.08$ & $4.38 \pm 1.06$ & 0.585 \\
\hline LDL-C (mmol/L) & $2.55 \pm 0.95$ & $2.60 \pm 0.83$ & 0.636 \\
\hline HDL-C (mmol/L) & $1.05 \pm 0.27$ & $1.14 \pm 0.28$ & $0.004 *$ \\
\hline AHI (times/h) & $28.60(21.00-42.80)$ & $2.60(1.65-3.50)$ & $<0.001 * *$ \\
\hline Lowest SpO2 (\%) & $80.88 \pm 11.08$ & $90.42 \pm 2.67$ & $<0.001 * *$ \\
\hline ESS & $6.00(2.00-10.00)$ & $3.00(1.00-6.00)$ & $0.018 *$ \\
\hline
\end{tabular}

Results are expressed as mean \pm standard deviation, median (interquartile range), or $n(\%)$. Differences between groups were analyzed by independent Student $t$ test, Fisher's exact test, $\chi^{2}$ text, or Wilcoxon test

$O S A$ obstructive sleep apnea, $B M I$ body mass index, $S B P$ systolic blood pressure, $D B P$ diastolic blood pressure, FPG fasting plasma glucose, $T G$ triglycerides, $T C$ total cholesterol, $L D L-C$ low-density lipoprotein cholesterol, $H D L-C$ high-density lipoprotein cholesterol, $A H I$ apnea-hypopnea index, ESS Epworth Sleepiness Scale $* P<0.05 ; * * P<0.001$

and fasting blood glucose (FBG; $P=0.201)$ between the two groups. Subjects with OSA had significantly higher BMIs and ESS $(P=0.018)$ than the non-OSA subjects $(P<0.001)$. Individuals with OSA also had higher diastolic blood pressure ( $P=0.032)$, triglycerides (TGs) $(P<0.001)$, AHI $(P<0.001)$, and lower SpO2 $(P<0.001)$ than control individuals. A greater proportion of individuals with OSA were male, obese, smokers, and had hypertension or dyslipidemia compared with the non-OSA group.

\section{PPARG sequencing and SNP detection}

We used a targeted next-generation sequencing approach to analyze PPARG gene sequences in 233 individuals with OSA and 93 controls. A total of 17 variants were identified in the PPARG sequence but only seven had a rs number. The details of the variants are shown in Supplemental Data Table S3. Among the seven PPARG variants, three were not represented in the 1000 Genomes Project database and were present in only one study subject. Three variants (rs1801282, rs3856806, and rs13073869) were single-nucleotide polymorphisms (SNPs) according to the 1000 Genomes Project database, whose minimum allele frequency is $\geq 0.01$. As shown in Supplemental Data Table S3, we also identified three rare non- synonymous variants (p.Y95C, p.V403A, and p.H30Y) which may be deleterious according to the SIFT and PolyPhen-2 databases. However, further study is required to determine the functions of these rare variants.

\section{Association of PPARG variants with the risk of having OSA}

Three SNPs in the PPARG sequence were identified in this study. Their allele frequency distributions are shown in Supplemental Data Table S4. All three SNPs were in Hardy-Weinberg equilibrium. The genotype frequencies at the rs1801282 SNP differed significantly $(P<0.05$, Table 2$)$ between the OSA and control group. The frequency of the $P P A R G$ rs1 $801282 \mathrm{G}$ allele was significantly lower in subjects with OSA $(4.72 \%)$ compared with subjects without OSA $\left(10.22 \% ; P^{\mathrm{a}}=0.027\right.$, Table 2). For the other two SNPS (rs3856806 and rs13073869), no significant differences were observed between the genotypes and allele distributions of the OSA and control groups. We also found that subjects with the PPARG rs1801282 CG genotype had decreased risk of having OSA compared with subjects with the CC genotype after adjusting for age, sex, BMI, smoking, alcohol consumption, TGs, high-density lipoprotein cholesterol (HDL-C), FBG, and 
Table 2 Genotype frequencies in OSA and non-OSA subjects

\begin{tabular}{llllll}
\hline SNPs & Genotype & OSA group (N/\%) N=233 & Non-OSA group (N/\%) sN=93 & $\chi^{2}$ & $P^{\mathrm{a}}$ \\
\hline rs1801282 & CC & $211(90.56 \%)$ & $74(79.57 \%)$ & 7.299 & $0.021^{*}$ \\
& CG & $22(9.44 \%)$ & $19(20.43 \%)$ & & \\
& C & $444(95.28 \%)$ & $167(89.78 \%)$ & 6.089 & $0.027^{*}$ \\
& G & $22(4.72 \%)$ & $19(10.22 \%)$ & & \\
rs3856806 & CC & $147(63.09 \%)$ & $51(54.84 \%)$ & & 0.337 \\
& CT & $80(34.33 \%)$ & $38(40.86 \%)$ & & \\
& TT & $6(2.58 \%)$ & $4(4.30 \%)$ & & \\
& C & $374(80.26 \%)$ & $140(75.27 \%)$ & & 0.194 \\
& T & $92(19,74 \%)$ & $46(24.73 \%)$ & & \\
rs13073869 & GG & $96(41.20 \%)$ & $41(44.09 \%)$ & & 0.111 \\
& GA & $116(49.79 \% \%)$ & $36(38.71 \%)$ & & \\
& AA & $21(9.01 \%)$ & $16(17.20 \%)$ & & \\
& G & $308(66.09 \%)$ & $118(63.44 \%)$ & & \\
& A & $158(33.91 \%)$ & $68(36.56 \%)$ & & \\
\hline
\end{tabular}

Results are expressed as $n(\%)$. Differences between groups were analyzed by $\chi^{2}$ text or Fisher's exact $O S A$ obstructive sleep apnea, $S N P s$ single nucleotide polymorphisms, $P^{\text {a }} P$ value was adjusted by step-down Bonferroni

$* P<0.05$

ESS $\left(\mathrm{OR}=0.318,95 \%\right.$ CI $\left.0.136-0.746 ; P^{\mathrm{a}}=0.008\right)$ (Table 3). No significant differences between the two groups were detected for the other two polymorphisms after adjustment for confounders.

Obesity is the most important risk factor for OSA and PPARG is related to obesity. To test if PPARG genotype was associated with OSA independent of obesity, obesity was stratified (Table 4). We found that obese subjects with the $P P A R G$ rs 1801282 CG genotype had decreased risk of having OSA compared with subjects with the CC genotype after adjusting for confounding factors $(\mathrm{OR}=0.166,95 \% \mathrm{CI}$ $0.030-0.901, P=0.037)$. Non-obese subjects with the PPARG rs1801282 CG genotype also had decreased risk of having OSA compared with subjects with the CC genotype after adjusting for confounding factors $(\mathrm{OR}=0.345,95 \% \mathrm{CI}$ $0.124-0.959, P=0.039)$. The risk of rs 1801282 to obesity was also analyzed in this study; we found that after adjusting for confounding factors, there were no differences between subjects with PPARG rs 1801282 CC genotype and CG genotype about risk of obesity in all subjects, OSA subjects, and non-OSA subjects in this study (Supplemental Data Table S5).

\section{Discussion}

The relationship between PPARG polymorphisms and OSA was investigated in individuals with and without OSA. Our results showed that the PPARG rs1801282 polymorphism was independently associated with OSA.

OSA is a complex disease that is influenced by a combination of genetic $[12,30]$ and environmental factors. The molecular mechanisms underlying OSA are not well understood. From a pathological viewpoint, several factors can cause OSA, including sympathetic nervous system activity, obesity, upper airway dilator muscle dysfunction, craniofacial abnormalities, heightened chemosensitivity, and a low arousal threshold $[9,31]$. These pathological processes are also influenced by genes.

PPARG is known to affect obesity, adipose and muscle tissue metabolism, and craniofacial abnormalities thereby causing OSA. Obesity is the strongest risk factor for the development of OSA [14], and PPARG plays an important role in the development of obesity $[15,16]$. PPARG regulates lipid and glucose metabolism, and downregulation of PPARG has been shown to have anti-obesity effects [32]. PPARG activation decreased free fatty acid levels and increased lipid storage in adipose tissue [33]. It was reported that craniofacial abnormalities and dysfunction of adipose and muscle tissue metabolism may cause upper airway obstruction, which is another risk factor for OSA. Roles of $P P A R G$ in regulating human adipocyte differentiation and adipogenesis have been identified [17]. Chang et al. [34] found that the progression of myogenesis was regulated by the level of intracellular PPARG. PPARG polymorphisms may be involved in the growth hormone/STAT5B pathway [35], and mice that were deficient in members of this pathway developed craniofacial abnormalities [30]. The growth hormone/STAT5B pathway also plays an important role in regulating energy metabolism in adipose and muscle tissue, suggesting that PPARG may cause craniofacial abnormalities and dysfunction of adipose and muscle tissue metabolism through this pathway $[36,37]$. In addition, the rs1801282 SNP is located in an exon and is a missense variant that affects the amino acid sequence of the PPARG protein 
Table 3 Multivariate logistic regression analyses of three SNPs in PPARG gene with the risk of OSA in all subjects

\begin{tabular}{|c|c|c|c|c|c|c|c|}
\hline \multirow[t]{2}{*}{ SNPs } & \multirow[t]{2}{*}{ Genotype } & \multicolumn{2}{|l|}{ unadjusted } & \multicolumn{2}{|l|}{ Model 1} & \multicolumn{2}{|l|}{ Model 2} \\
\hline & & OR (95CI) & $P^{\mathrm{a}}$ & OR (95CI) & $P^{\mathrm{a}}$ & OR (95CI) & $P^{\mathrm{a}}$ \\
\hline \multirow[t]{2}{*}{ rs 1801282} & $\mathrm{CC}(N=285)$ & 1 & & 1 & & 1 & \\
\hline & $\mathrm{CG}(N=41)$ & $0.406(0.208-0.792)$ & $0.040 *$ & $0.278(0.126-0.612)$ & $0.005^{*}$ & $0.318(0.136-0.746)$ & $0.008 *$ \\
\hline \multirow[t]{3}{*}{ rs 3856806} & $\mathrm{CC}(N=198)$ & 1 & & 1 & & 1 & \\
\hline & $\mathrm{CT}(N=118)$ & $0.730(0.443-1.205)$ & 0.657 & $1.393(0.731-2.654)$ & 0.626 & $0.898(0.479-1.683)$ & 0.898 \\
\hline & $\mathrm{TT}(N=10)$ & $0.520(0.141-1.918)$ & 0.326 & $0.635(0.234-1.726)$ & 0.373 & $0.684(0.125-3.737)$ & 0.684 \\
\hline \multirow[t]{3}{*}{ rs 13073869} & $\mathrm{GG}(N=137)$ & 1 & & 1 & & 1 & \\
\hline & $\mathrm{GA}(N=152)$ & $1.376(0.816-2.322)$ & 0.462 & $1.378(0.752-2.523)$ & 0.897 & $1.643(0.856-3.153)$ & 0.136 \\
\hline & $\mathrm{AA}(N=37)$ & $0.561(0.266-1.182)$ & 0.512 & $0.552(0.230-1.324)$ & 0.732 & $0.654(0.258-1.657)$ & 0.371 \\
\hline
\end{tabular}

Model 1: adjusted for age, sex, BMI, smoker and drinker. Model 2: adjusted for model 1 + TG, HDL-C, FBG and ESS

$S N P s$ single nucleotide polymorphisms, $P P A R G$ peroxisome proliferator-activated receptor gamma, $O S A$ obstructive sleep apnea, $B M I$ body mass index, $T G$ triglycerides, $H D L-C$ high-density lipoprotein cholesterol, $F P G$ fasting plasma glucose, ESS Epworth Sleepiness Scale, $P^{\mathrm{a}} P$ value was adjusted by step-down Bonferroni

$* P<0.05$

(Pro12Ala). Together, these findings indicated that variants in PPARG may affect the development of OSA, although the exact mechanism remains unclear.

We found that the frequency of the PPARG G allele was significantly lower in subjects with OSA compared with subjects without OSA. This association was present both in obese and non-obese individuals with OSA. Bharat et al. previously found that the frequency of the PPARG $\mathrm{G}$ allele was significantly higher in subjects with OSA when compared to obese control [19]. It was reported that allele subtypes differed in lean, overweight, and obese subjects [38]. However, the results of our study were inconsistent with those of Bharat et al. in obese subjects. All of the individuals included in our study were Chinese Han while the individuals studied by Bharat et al. were Asian Indians. This further illustrates the genetic differences at the PPARG locus between different ethnic groups.

We also found that rs1801282 was associated with OSA, whereas previously, Guan et al. [20] found no association between rs1801282 and OSA in a Chinese Han population. We found that the Guan et al.'s study was designed as a case-control and our study was a cross-sectional study, which may be the reasons that there were differences between Guan et al.'s study and our study. We hypothesized that genes linked with metabolic disorders may be relevant to the genetics of OSA because of the prominence of metabolic disorders in the syndrome's phenotype. The PPARG rs1801282 polymorphism has attracted much attention because of its correlation with various metabolic conditions including obesity, diabetes, and dyslipidemia. In this study, we confirmed that the PPARG rs1801282 polymorphism was associated with OSA.

Previous studies showed that age was an independent risk factor for OSA [9] and that most individuals with OSA were male [9]. In our study, there were more male individuals in the OSA group than in the non-OSA group, so to avoid confounding effects, we adjusted for age and sex. Obesity plays an important role in OSA. PPARG rs1801282 polymorphisms were associated with decreased BMI and may slightly protect against childhood

Table 4 Multivariate logistic regression analyses of rs 1801282 with the risk of OSA in stratified analysis according to obesity

\begin{tabular}{|c|c|c|c|c|c|c|c|}
\hline & \multirow[t]{2}{*}{ Genotype } & \multicolumn{2}{|l|}{ Unadjusted } & \multicolumn{2}{|l|}{ Model 1} & \multicolumn{2}{|l|}{ Model 2} \\
\hline & & OR (95CI) & $P$ value & OR (95CI) & $P$ value & OR (95CI) & $P$ value \\
\hline \multirow{2}{*}{$\begin{array}{l}\text { Obesity } \\
N=114\end{array}$} & $\mathrm{CC}$ & 1 & & 1 & & 1 & \\
\hline & $\mathrm{CG}$ & $0.188(0.056-0.628)$ & $0.007 *$ & $0.150(0.039-0.580)$ & $0.006^{*}$ & $0.166(0.030-0.901)$ & $0.037 *$ \\
\hline \multirow{2}{*}{$\begin{array}{l}\text { Non-obesity } \\
N=212\end{array}$} & $\mathrm{CC}$ & 1 & & 1 & & 1 & \\
\hline & $\mathrm{CG}$ & $0.447(0.190-1.054)$ & 0.066 & $0.368(0.139-0.977)$ & $0.045^{*}$ & $0.345(0.124-0.959)$ & $0.041 *$ \\
\hline
\end{tabular}

Model 1: adjusted for age, sex, BMI, smoker and drinker. Model 2: adjusted for model $1+$ TG, HDL-C, FBG and ESS

$O S A$ obstructive sleep apnea, $B M I$ body mass index, $T G$ triglycerides, $H D L-C$ high-density lipoprotein cholesterol, $F P G$ fasting plasma glucose, ESS Epworth Sleepiness Scale

$* P<0.05$ 
obesity [39]. BMI is associated with PPARG and adipogenesis is affected by PPARG [19], a known regulator of lipid and glucose metabolism. We found that individuals with OSA had higher BMI and TGs but lower HDL-C than non-OSA subjects. To avoid confounding effects, we also adjusted for BMI, TGs, HDL-C, and FBG.

Care was taken to avoid bias in this study. Genomic DNA extraction and targeted sequencing were performed according to the manufacturer's instructions by a trained experimentalist who was unaware of the subjects' clinical status. In the statistical analysis, adjustments were made for the confounding effects of risk factors for OSA and PPARG. Finally, the cross-sectional design of the study and consecutive recruitment of subjects reduced the effects of outcome-selection bias.

Despite this, our study had some limitations. First, the sample size was small and larger studies are needed to confirm our results. Second, the participants may not be entirely representative of the general Han Chinese population. Potential falsepositive results may still be possible after multiple corrections. Prospective cohort studies are needed to confirm the variants and associations identified in our study. Third, the controls of this study were also from Otolaryngological Department but not from the population. Finally, the exact mechanisms of the identified PPARG variants is not fully understood and requires further functional studies.

In conclusion, we identified a $P P A R G$ variant in patients with OSA. The variant was predicted to be associated with the occurrence of OSA in Chinese Han subjects. Individuals with the PPARG rs1801282 CG genotype were at lower risk of having OSA than individuals with the $\mathrm{CC}$ genotype after adjusting for confounding effects. Genetic analysis may be helpful for individualized typing of patients with OSA and could contribute to personalized diagnosis and treatment.

Acknowledgments The authors thank all the participants in this study. We also thank Qianlan Zhong for her help during the data analysis. Finally, we thank Margaret Biswas, PhD, from Liwen Bianji, Edanz Group China (www.liwenbianji.cn/ac), for editing the English text of a draft of this manuscript.

Authors' contributions XLJ performed the experiments. YYY and SY prepared the human samples. JL performed the data analysis. YWQ designed the study and prepared the manuscript. All the authors read and approved the final manuscript.

Funding This study was supported by the National Natural Science Foundation of China (Grant Nos. 81670331, 91439127), Beijing Natural Science Foundation (Grant No.7192030, 7172040), Beijing Municipal Health Commission Finance Project (2019-0026), Beijing Billion Talent Project (2017-A-10), Beijing Medical Project (Grant No. 2016 4), the Beijing Key Laboratory of Upper Airway Dysfunction and Related Cardiovascular Diseases (No: BZ0377), International Science and Technology Cooperation Program of China (Grant No. 2015DFA30160), and the Chinese Ministry of Science and Technology (Grant No. 2012CB945104). The research funds were used to design the study, prepare human samples, and perform targeted sequencing.

\section{Compliance with ethical standards}

Ethics approval and consent to participate This study was approved by the Medicine Ethics Committee of Beijing Anzhen Hospital and registered in the Chinese Clinical Trial Registry (No. ChiCTR-ROC17011027).

Consent for publication All participants provided written informed consent before enrollment in this study.

Availability of data and materials All data generated or analyzed during this study are included in this published article and its supplementary information files. Data are freely available to any scientist who wishes to use them without breaching participant confidentiality.

Competing interests The authors declare that they have no competing interests.

Abbreviations OSA, Obstructive sleep apnea; PPARG, Peroxisome proliferator-activated receptor gamma; SNP, Single nucleotide polymorphism; AHI, Apnea-hypopnea index; BMI, Body mass index; TG, Triglyceride; HDL-C, High-density lipoprotein cholesterol; FBG, Fasting blood glucose

Open Access This article is distributed under the terms of the Creative Commons Attribution 4.0 International License (http:// creativecommons.org/licenses/by/4.0/), which permits unrestricted use, distribution, and reproduction in any medium, provided you give appropriate credit to the original author(s) and the source, provide a link to the Creative Commons license, and indicate if changes were made.

\section{References}

1. Cade BE, Chen H, Stilp AM, Gleason KJ, Sofer T, Ancoli-Israel S, Arens R, Bell GI, Below JE, Bjonnes AC, Chun S, Conomos MP, Evans DS, Johnson WC, Frazier-Wood AC, Lane JM, Larkin EK, Loredo JS, Post WS, Ramos AR, Rice K, Rotter JI, Shah NA, Stone KL, Taylor KD, Thornton TA, Tranah GJ, Wang C, Zee PC, Hanis CL, Sunyaev SR, Patel SR, Laurie CC, Zhu X, Saxena R, Lin X, Redline S (2016) Genetic associations with obstructive sleep apnea traits in Hispanic/Latino Americans. Am J Respir Crit Care Med 194:886-897

2. Peppard PE, Young T, Barnet JH, Palta M, Hagen EW, Hla KM (2013) Increased prevalence of sleep-disordered breathing in adults. Am J Epidemiol 177:1006-1014

3. Chen X, Wang R, Zee P, Lutsey PL, Javaheri S, Alcantara C, Jackson CL, Williams MA, Redline S (2015) Racial/ethnic differences in sleep disturbances: the multi-ethnic study of atherosclerosis (MESA). Sleep 38:877-888

4. Leung RS, Bradley TD (2001) Sleep apnea and cardiovascular disease. Am J Respir Crit Care Med 164:2147-2165

5. Strohl KP, Saunders NA, Feldman NT, Hallett M (1978) Obstructive sleep apnea in family members. N Engl J Med 299: 969-973

6. Palmer LJ, Buxbaum SG, Larkin E, Patel SR, Elston RC, Tishler PV, Redline S (2003) A whole-genome scan for obstructive sleep apnea and obesity. Am J Hum Genet 72:340-350

7. Kripke DF, Kline LE, Nievergelt CM, Murray SS, Shadan FF, Dawson A, Poceta JS, Cronin J, Jamil SM, Tranah GJ, Loving RT, Grizas AP, Hahn EK (2015) Genetic variants associated with 
sleep disorders. Sleep Med 16:217-224

8. Varvarigou V, Dahabreh IJ, Malhotra A, Kales SN (2011) A review of genetic association studies of obstructive sleep apnea: field synopsis and meta-analysis. Sleep 34:1461-1468

9. Jordan AS, McSharry DG, Malhotra A (2014) Adult obstructive sleep apnoea. Lancet 383:736-747

10. Palmer LJ, Buxbaum SG, Larkin EK, Patel SR, Elston RC, Tishler PV, Redline S (2004) Whole genome scan for obstructive sleep apnea and obesity in African-American families. Am J Respir Crit Care Med 169:1314-1321

11. Carmelli D, Colrain IM, Swan GE, Bliwise DL (2004) Genetic and environmental influences in sleep-disordered breathing in older male twins. Sleep 27:917-922

12. Zhang D, Xiao Y, Luo J (2014) Genetics of obstructive sleep apnea/ hypopnea syndrome. Chin Med J 127:3135-3141

13. Redline S, Tishler PV, Tosteson TD, Williamson J, Kump K, Browner I, Ferrette V, Krejci P (1995) The familial aggregation of obstructive sleep apnea. Am J Respir Crit Care Med 151:682-687

14. Tuomilehto H, Seppa J, Uusitupa M (2013) Obesity and obstructive sleep apnea-clinical significance of weight loss. Sleep Med Rev 17: 321-329

15. Lehrke M, Lazar MA (2005) The many faces of PPARgamma. Cell 123:993-999

16. Aranaz P, Romo-Hualde A, Zabala M, Navarro-Herrera D, Ruiz de Galarreta M, Gil AG, Martinez JA, Milagro FI, Gonzalez-Navarro CJ (2017) Freeze-dried strawberry and blueberry attenuates dietinduced obesity and insulin resistance in rats by inhibiting adipogenesis and lipogenesis. Food Funct 8:3999-4013

17. Janani C, Ranjitha Kumari BD (2015) PPAR gamma gene-a review. Diabetes Metab Syndr 9:46-50

18. Gharib SA, Hayes AL, Rosen MJ, Patel SR (2013) A pathwaybased analysis on the effects of obstructive sleep apnea in modulating visceral fat transcriptome. Sleep 36:23-30

19. Bhushan B, Guleria R, Misra A, Luthra K, Kumar G (2011) Association of PPARgamma2 (Pro12Ala) and neuropeptide Y (Leu7Pro) gene polymorphisms with obstructive sleep apnea in obese Asian Indians. Dis Markers 30:31-38

20. Guan J, Yi H, Wu X, Su K, Tao M, Yin S (2011) Pro12Ala polymorphism in human peroxisome proliferator activated receptor gamma is associated with hyperlipidaemia in obstructive sleep apnoea hypopnoea syndrome. J Laryngol Otol 125:1042-1048

21. Gottlieb DJ, Punjabi NM, Mehra R, Patel SR, Quan SF, Babineau DC, Tracy RP, Rueschman M, Blumenthal RS, Lewis EF, Bhatt DL, Redline S (2014) CPAP versus oxygen in obstructive sleep apnea. N Engl J Med 370:2276-2285

22. Kushida CA, Nichols DA, Quan SF, Goodwin JL, White DP, Gottlieb DJ, Walsh JK, Schweitzer PK, Guilleminault C, Simon RD, Leary EB, Hyde PR, Holmes TH, Bloch DA, Green S, McEvoy LK, Gevins A, Dement WC (2006) The apnea positive pressure long-term efficacy study (APPLES): rationale, design, methods, and procedures. J Clin Sleep Med 2:288-300

23. Budhiraja R, Kushida CA, Nichols DA, Walsh JK, Simon RD, Gottlieb DJ, Quan SF (2017) Predictors of sleepiness in obstructive sleep apnoea at baseline and after 6 months of continuous positive airway pressure therapy. Eur Respir J 50:1700348

24. Berry RB, Budhiraja R, Gottlieb DJ, Gozal D, Iber C, Kapur VK, Marcus CL, Mehra R, Parthasarathy S, Quan SF, Redline S, Strohl KP, Davidson Ward SL, Tangredi MM, American Academy of Sleep M (2012) Rules for scoring respiratory events in sleep: update of the 2007 AASM manual for the scoring of sleep and associated events. Deliberations of the Sleep Apnea Definitions Task Force of the American Academy of Sleep Medicine. J Clin Sleep Med 8: 597-619

25. Myers KA, Mrkobrada M, Simel DL (2013) Does this patient have obstructive sleep apnea?: the rational clinical examination systematic review. JAMA 310:731-741
26. Johnson DA, Thomas SJ, Na Guo MA, Yano Y, Rueschman M, Tanner RM, Mittleman MA, Calhoun DA, Wilson JG, Muntner P, Redline S (2018) Association between sleep apnea and blood pressure control among blacks: Jackson heart sleep study. Circulation undefined:undefined

27. Cheung YY, Tai BC, Loo G, Khoo SM, Cheong KY, Barbe F, Lee $\mathrm{CH}$ (2017) Screening for obstructive sleep apnea in the assessment of coronary risk. Am J Cardiol 119:996-1002

28. Jiao X, He J, Yang Y, Yang S, Li J, Qin Y (2018) Associations between circulating full-length angiopoietin-like protein 8 levels and severity of coronary artery disease in Chinese non-diabetic patients: a case-control study. Cardiovasc Diabetol 17:92

29. Zhou BF (2002) Effect of body mass index on all-cause mortality and incidence of cardiovascular diseases-report for meta-analysis of prospective studies open optimal cut-off points of body mass index in Chinese adults. Biomed Environ Sci 15:245-252

30. Redline S, Tishler PV (2000) The genetics of sleep apnea. Sleep Med Rev 4:583-602

31. Campana L, Eckert DJ, Patel SR, Malhotra A (2010) Pathophysiology \& genetics of obstructive sleep apnoea. Indian J Med Res 131:176-187

32. Imam MU, Ismail M, Ithnin H, Tubesha Z, Omar AR (2013) Effects of germinated brown rice and its bioactive compounds on the expression of the peroxisome proliferator-activated receptor gamma gene. Nutrients 5:468-477

33. Kim K, Boo K, Yu YS, Oh SK, Kim H, Jeon Y, Bhin J, Hwang D, Kim KI, Lee JS, Im SS, Yoon SG, Kim IY, Seong JK, Lee H, Fang S, Baek SH (2017) RORalpha controls hepatic lipid homeostasis via negative regulation of PPARgamma transcriptional network. Nat Commun 8:162

34. Chang YJ, Chen YJ, Huang CW, Fan SC, Huang BM, Chang WT, Tsai YS, Su FC, Wu CC (2016) Cyclic stretch facilitates myogenesis in $\mathrm{C} 2 \mathrm{C} 12$ myoblasts and rescues thiazolidinedioneinhibited myotube formation. Front Bioeng Biotechnol 4:27

35. Chen D, Jin G, Wang Y, Wang H, Liu H, Liu Y, Fan W, Ma H, Miao R, Hu Z, Sun W, Qian J, Jin L, Wei Q, Shen H, Huang W, Lu D (2008) Genetic variants in peroxisome proliferator-activated receptor-gamma gene are associated with risk of lung cancer in a Chinese population. Carcinogenesis 29:342-350

36. Vendelbo MH, Christensen B, Gronbaek SB, Hogild M, Madsen M, Pedersen SB, Jorgensen JO, Jessen N, Moller N (2015) GH signaling in human adipose and muscle tissue during 'feast and famine': amplification of exercise stimulation following fasting compared to glucose administration. Eur J Endocrinol 173:283-290

37. Futawaka K, Tagami T, Fukuda Y, Koyama R, Nushida A, Nezu S, Imamoto M, Kasahara M, Moriyama K (2016) Growth hormone regulates the expression of UCP2 in myocytes. Growth Hormon IGF Res 29:57-62

38. Hsiao TJ, Lin E (2015) The Pro12Ala polymorphism in the peroxisome proliferator-activated receptor gamma (PPARG) gene in relation to obesity and metabolic phenotypes in a Taiwanese population. Endocrine 48:786-793

39. Almeida SM, Furtado JM, Mascarenhas P, Ferraz ME, Ferreira JC, Monteiro MP, Vilanova M, Ferraz FP (2018) Association between LEPR, FTO, MC4R, and PPARG-2 polymorphisms with obesity traits and metabolic phenotypes in school-aged children. Endocrine 60:466-478

Publisher's note Springer Nature remains neutral with regard to jurisdictional claims in published maps and institutional affiliations. 